\title{
ESTIMATES FOR MIXED CHARACTER SUMS
}

\author{
NICHOLAS M. KATZ
}

\section{INTRODUCTION}

Let $k$ be a finite field, $p$ its characteristic, and

$$
\psi:(k,+) \rightarrow \mathbb{Z}\left[\zeta_{p}\right]^{\times} \subset \mathbb{C}^{\times}
$$

a nontrivial additive character of $k$. We are given a polynomial $f=$ $f\left(x_{1}, \ldots, x_{n}\right)$ in $n \geq 1$ variables over $k$ of degree $d \geq 1$ which is a "Deligne polynomial", i.e., its degree $d$ is prime to $p$ and its highest degree term, say $f_{d}$, is a homogeneous form of degree $d$ in $n$ variables which is nonzero, and whose vanishing, if $n \geq 2$, defines a smooth hypersurface in the projective space $\mathbb{P}^{n-1}$. For a Deligne polynomial $f$ as above, one has Deligne's fundamental estimate [De-Weil I, 8.4]

$$
\left|\sum_{x \in k^{n}} \psi(f(x))\right| \leq(d-1)^{n} \#(k)^{n / 2} .
$$

Suppose now that for each index $1 \leq i \leq n$ we are given a $\mathbb{C}$-valued function on $k, x \mapsto \mathcal{G}_{i}(x)$, and we are interested in good (i.e., square root cancellation) estimates for the sum

$$
\sum_{\left(x_{1}, \ldots, x_{n}\right) \in k^{n}} \psi\left(f\left(x_{1}, \ldots, x_{n}\right)\right) \prod_{i=1}^{n} \mathcal{G}_{i}\left(x_{i}\right) .
$$

We will give such estimates if the Deligne polynomial $f=f\left(x_{1}, \ldots, x_{n}\right)$ is nicely adapted to the particular choice of coordinates $\left(x_{1}, \ldots, x_{n}\right)$, and if each of the functions $x \mapsto \mathcal{G}_{i}(x)$ is suitably algebro-geometric in nature.

The relevant notion of $f$ 's being nicely adapted to the particular choice of coordinates is that $f$ be Dwork-regular. We say that a Deligne polynomial $f=f\left(x_{1}, \ldots, x_{n}\right)$ is Dwork-regular if, for every proper subset $S \subset\{1,2, \ldots, n\}$, the polynomial obtained by specializing $x_{i} \mapsto 0$ for $i \in S$ is a Deligne polynomial of the same degree $d$ in the remaining variables $x_{j}, j \notin S$. The archtypical example of a Dwork-regular $f$ is $f=\sum_{i=1}^{n} x_{i}^{d}+$ (terms of lower degree). The antithesis of a Dworkregular $f$ is, in any odd characteristic, the split quadratic form in an even number $n=2 m$ of variables $f=\sum_{i=1}^{m} x_{i} x_{m+i}$. 
Explaining in general what we require of the functions $x \mapsto \mathcal{G}_{i}(x)$, and stating the general result, will require familiarity with $\ell$-adic cohomology. However, various special cases of our result can be formulated, if not proven, in entirely elementary terms. As already these special cases may be useful to number theorists, we first make explicit these elementary formulations in three example situations.

We begin with the example of multiplicative characters. We adopt the convention of extending multiplicative characters (even trivial ones) $\chi$ of $k^{\times}$to $k$ by defining

$$
\chi(0)=0 .
$$

Suppose for each index $i$ we are given a multiplicative character $\chi_{i}$ : $k^{\times} \rightarrow \mathbb{C}^{\times}$, and a non-zero one variable polynomial $p_{i}(x) \in k[x]$. We suppose that the following nondegeneracy condition holds for each $i$ : for every zero $\alpha \in \bar{k}$ of $p_{i}$, denoting by $\operatorname{ord}_{\alpha}\left(p_{i}\right)$ the multiplicity of $\alpha$ as a zero of $p_{i}$, the multiplicative character $\chi_{i}^{\text {ord }}\left(p_{i}\right)$ is nontrivial. [For instance, this is automatic if $p_{i}$ is a nonzero constant, or if $\chi_{i}$ is nontrivial and $p_{i}$ is squarefree.] Then we take for $x \mapsto \mathcal{G}_{i}(x)$ the function on $k$ given by

$$
x \mapsto \chi_{i}\left(p_{i}(x)\right) .
$$

Theorem 1.1. Suppose the Deligne polynomial $f=f\left(x_{1}, \ldots, x_{n}\right)$ of prime-to- $p$ degree $d$ is Dwork-regular, and that the data $\left(\chi_{i}, p_{i}\right)_{i}$ satisfies the above nondegeneracy conditions. For each $i$, denote by $e_{i}$ the number of distinct zeroes of $p_{i}$ in $\bar{k}$. Then we have the estimate

$$
\left|\sum_{\left(x_{1}, \ldots, x_{n}\right) \in k^{n}} \psi\left(f\left(x_{1}, \ldots, x_{n}\right)\right) \prod_{i=1}^{n} \chi_{i}\left(p_{i}\left(x_{i}\right)\right)\right| \leq\left(\prod_{i=1}^{n}\left(d-1+e_{i}\right)\right) \#(k)^{n / 2} .
$$

Remark 1.2. Here is a simple example to show why we must assume the Deligne polynomial $f$ to be Dwork-regular, already with $n=2$. Suppose $\mathrm{p}$ is odd, and take $d=2, f=x_{1} x_{2}$. Choose a nontrivial multiplicative character $\chi$, and for both $i=1,2$, take $\left(\chi_{i}, p_{i}\right)=\left(\chi, x_{i}\right)$. Then the sum in question is

$$
\sum_{\left(x_{1}, x_{2}\right) \in k^{2}} \psi\left(x_{1} x_{2}\right) \chi\left(x_{1} x_{2}\right)=(\# k-1) G(\psi, \chi),
$$

with $G(\psi, \chi)$ the Gauss sum $\sum_{t \in k} \psi(t) \chi(t)$. So for growing \#k, the sum is $O\left(\# k^{3 / 2}\right)$ but is not the desired $O\left(\# k^{2 / 2}\right)$.

We next consider the example of additive characters. We extend the additive character $\psi$ of $k$ to $\mathbb{P}^{1}(k)=k \cup\{\infty\}$ by defining

$$
\psi(\infty)=0 \text {. }
$$


Suppose that for each index $i$ we are given a one variable rational function $r_{i}(x) \in k(x)$, which we assume satisfies the following two nondegeneracy conditions, one on the finite poles, if any, of $r_{i}$, and one on the pole, if any, of $r_{i}$ at $\infty$ :

(1) For each $\beta \in \bar{k}$, the multiplicity $\operatorname{poleord}_{\beta}\left(r_{i}\right)$ of $\beta$ as a pole of $r_{i}$ is either prime-to-p or 0 .

(2) At $\infty$, we require that either poleord $d_{\infty}\left(r_{i}\right)<d$ or that poleord $d_{\infty}\left(r_{i}\right)$ is both prime-to-p and $>d$.

Then we take for $x \mapsto \mathcal{G}_{i}(x)$ the function on $k$ given by

$$
x \mapsto \psi\left(r_{i}(x)\right) .
$$

Theorem 1.3. Suppose the Deligne polynomial $f=f\left(x_{1}, \ldots, x_{n}\right)$ of prime-to-p degree $d$ is Dwork-regular, and the data $\left(r_{i}\right)_{i}$ satisfies the above nondegeneracy conditions. For each $i$, denote by $m_{i}$ the sum

$$
m_{i}:=\sum_{\text {poles }} \sum_{\beta \in \bar{k}}\left(1+\text { poleord }_{\beta}\left(r_{i}\right)\right),
$$

and by $d_{i}$ the integer

$$
d_{i}=\operatorname{Max}\left(d, \operatorname{poleord}_{\infty}\left(r_{i}\right)\right) .
$$

Then we have the estimate

$$
\left|\sum_{\left(x_{1}, \ldots, x_{n}\right) \in k^{n}} \psi\left(f\left(x_{1}, \ldots, x_{n}\right)\right) \prod_{i=1}^{n} \psi_{i}\left(r_{i}\left(x_{i}\right)\right)\right| \leq\left(\prod_{i=1}^{n}\left(d_{i}-1+m_{i}\right)\right) \#(k)^{n / 2} .
$$

Remark 1.4. The reason we don't allow poleord $d_{\infty}\left(r_{i}\right)=d$ is that already in one variable, we have $\sum_{x \in k} \psi(f(x)) \mathcal{G}(x)=\# k$ for $\mathcal{G}(x)=$ $\psi(-f(x))$.

As our final example situation, we take a mixture of additive and multiplicative characters, which includes both of the previous example situations as special cases. For each index $i$ we are given a multiplicative character $\chi_{i}$, a one-variable polynomial $p_{i}$, and a one-variable rational function $r_{i}$. We suppose that for each $i$ the triple $\left(\chi_{i}, p_{i}, r_{i}\right)$ satisfies the following nondegeneracy conditions: the rational function $r_{i}$ satisfies the nondegeneracy conditions given in the additive character exampe, and for every zero $\alpha \in \bar{k}$ of $p_{i}$ which is not a pole of $r_{i}$, $\chi_{i}^{\text {ord }_{\alpha}\left(p_{i}\right)}$ is nontrivial. [But there is no condition imposed at zeros of $p_{i}$ which are also poles of $r_{i}$.] Then we take for $x \mapsto \mathcal{G}_{i}(x)$ the function on $k$ given by

$$
x \mapsto \psi\left(r_{i}(x)\right) \chi_{i}\left(p_{i}(x)\right)
$$


Theorem 1.5. Suppose the Deligne polynomial $f=f\left(x_{1}, \ldots, x_{n}\right)$ of prime-to-p degree $d$ is Dwork-regular, and the data $\left(\chi_{i}, p_{i}, r_{i}\right)_{i}$ satisfies the above nondegeneracy conditions. For each $i$, denote by $m_{i}$ the sum

$$
m_{i}:=\sum_{\text {poles }} \sum_{\beta \in \bar{k} \text { of } r_{i}}\left(1+\text { poleord }_{\beta}\left(r_{i}\right)\right),
$$

denote by $d_{i}$ the integer

$$
d_{i}=\operatorname{Max}\left(d, \text { poleord }_{\infty}\left(r_{i}\right)\right),
$$

and denote by $e_{i, n o p o l e}$ the number of distinct zeroes of $p_{i}$ in $\bar{k}$ which are not poles of $r_{i}$. Then we have the estimate

$$
\begin{gathered}
\left|\sum_{\left(x_{1}, \ldots, x_{n}\right) \in k^{n}} \psi\left(f\left(x_{1}, \ldots, x_{n}\right)\right) \prod_{i=1}^{n}\left(\psi\left(r_{i}\left(x_{i}\right)\right) \chi_{i}\left(p_{i}\left(x_{i}\right)\right)\right)\right| \\
\leq\left(\prod_{i=1}^{n}\left(d_{i}-1+m_{i}+e_{i, \text { nopole }}\right)\right) \#(k)^{n / 2} .
\end{gathered}
$$

Remark 1.6. Here is a useful mnemonic to remember the statement of the theorem; it is as though the variables in the Dwork-regular $f$ separate completely. Indeed, if $f\left(x_{1}, \ldots, x_{n}\right)=\sum_{i=1}^{n} f_{i}\left(x_{i}\right)$, for onevariable polynomials $f_{i}$, each of degree $d$, then the sum being estimated is the product of one variable sums, and the asserted estimate is the product of the standard estimates for such one-variable sums, which go back to Weil [We], along lines foreseen by Hasse [Ha].

Here is slight variant on the previous theorem, where we weaken slightly the hypothesis on $f$, but strengthen slightly the the hypotheses on the $\mathcal{G}_{i}$.

Theorem 1.7. Suppose $f=f\left(x_{1}, \ldots, x_{n}\right)$ is a polynomial of prime-to- $p$ degree $\leq d$ in $n$ variables (not necessarily a Deligne polynomial). If $n \geq 2$, suppose that the polynomial $f\left(x_{1}, \ldots, x_{n-1}, 0\right)$ obtained by specializing $x_{n} \mapsto 0$, is a Deligne polynomial of degree $d$ in $n-1$ variables which is Dwork-regular (as a polynomial in $n-1$ variables). Suppose that the data $\left(\chi_{i}, p_{i}, r_{i}\right)_{i}$ satisfies the above nondegeneracy conditions. Suppose in addition that the data $\left(\chi_{n}, p_{n}, r_{n}\right)$ attached to the last variable satisfies the following additional condition: either poleord $d_{\infty}\left(r_{i}\right)$ is both prime-to- $p$ and $>d$, or $r_{n}$ has a pole in $\bar{k}$, or $p_{n}$ has a zero in $\bar{k}$. Then the estimate of the previous theorem,

$$
\left|\sum_{\left(x_{1}, \ldots, x_{n}\right) \in k^{n}} \psi\left(f\left(x_{1}, \ldots, x_{n}\right)\right) \prod_{i=1}^{n}\left(\psi\left(r_{i}\left(x_{i}\right)\right) \chi_{i}\left(p_{i}\left(x_{i}\right)\right)\right)\right|
$$




$$
\leq\left(\prod_{i=1}^{n}\left(d_{i}-1+m_{i}+e_{i, \text { nopole }}\right)\right) \#(k)^{n / 2},
$$

remains valid.

Remark 1.8. Let us explain why we need the extra condition imposed on the $n$ 'th datum $\left(\chi_{n}, p_{n}, r_{n}\right)$. The "worst case" of the above theorem is arguably when $f\left(x_{1}, \ldots, x_{n}\right)=F\left(x_{1}, \ldots, x_{n-1}\right)$ does not involve the variable $x_{n}$ at all, and where $F$ is a Dwork-regular Deligne polynomial in $n-1$ variables. Then our sum is the product of the $n-1$ variable sum

$$
\sum_{\left(x_{1}, \ldots, x_{n-1}\right) \in k^{n-1}} \psi\left(F\left(x_{1}, \ldots, x_{n-1}\right)\right) \prod_{i=1}^{n-1}\left(\psi\left(r_{i}(x)\right) \chi_{i}\left(p_{i}\left(x_{i}\right)\right)\right)
$$

with the sum

$$
\sum_{x \in k} \psi\left(r_{n}(x)\right) \chi_{i}\left(p_{n}(x)\right)
$$

So here we must rule out the case when both $p_{n}$ and $r_{n}$ are constant, in order to have a good estimate for this last sum. And if, say, $f\left(x_{1}, \ldots, x_{n}\right)=F\left(x_{1}, \ldots, x_{n-1}\right)+f_{n}\left(x_{n}\right)$, with $f_{n}$ of some lower, prime-to-p degree $d_{n}<d$, then our sum is the product of the $n-1$ variable sum above with the one- variable sum

$$
\sum_{x \in k} \psi\left(f_{n}(x)+r_{n}(x)\right) \chi_{i}\left(p_{n}(x)\right)
$$

and we must rule out the case when $p_{n}$ is constant and $r_{n}$ is $-f_{n}$.

\section{Statement of the MAIN Results}

In this section, we continue to work over the finite field $k$, with the chosen nontrivial $\mathbb{C}$-valued additive character $\psi$, extended by zero to $\mathbb{P}^{1}(k)=k \cup\{\infty\}$. We choose a prime number $\ell \neq p$, and an isomorphism of fields $\iota: \mathbb{C} \cong \overline{\mathbb{Q}}_{\ell}$. This allows us to view $\mathbb{C}$-valued additive and multiplicative characters of $k$ as having values in $\overline{\mathbb{Q}}_{\ell}$, and to speak of the complex absolute values of elements in $\overline{\mathbb{Q}}_{\ell}$. Henceforth, we work entirely with $\overline{\mathbb{Q}}_{\ell}$-sheaves.

Given any separated scheme $X / k$ of finite type, any constructible $\overline{\mathbb{Q}}_{\ell^{-}}$sheaf $\mathcal{F}$ on $X / k$, any finite extension field $E / k$, and any point $x \in$ $X(E)$, we denote by

$$
\operatorname{Frob}_{E, x} \mid \mathcal{F}
$$

the action of the geometric Frobenius element (inverse of the standard generator) $\operatorname{Frob}_{E} \in \operatorname{Gal}(\bar{E} / E)$ on the pullback of $\mathcal{F}$ to $\operatorname{Spec}(E)$ by the 
point $x \in X(E)$, viewed as a morphism $x: \operatorname{Spec}(E) \rightarrow X$. We write

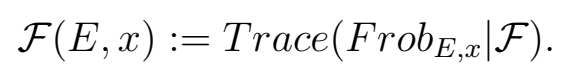

Attached to the constructible $\overline{\mathbb{Q}}_{\ell^{-}}$sheaf $\mathcal{F}$ on $X / k$ are its compact cohomology groups $H_{c}^{i}\left(X \otimes_{k} \bar{k}, \mathcal{F}\right)$. These are finite dimensional $\overline{\mathbb{Q}}_{\ell^{-}}$ spaces, endowed with an action of $\mathrm{Frob}_{k}$, which vanish except possibly for $0 \leq i \leq 2 \operatorname{dim}(X)$. The Lefschetz Trace formula [Gr-Rat] asserts that for any finite extension field $E / k$, we have

$$
\sum_{x \in X(E)} \mathcal{F}(E, x)=\sum_{i}(-1)^{i} \operatorname{Trace}\left(\operatorname{Frob}_{E} \mid H_{c}^{i}\left(X \otimes_{k} \bar{k}, \mathcal{F}\right)\right) .
$$

We now turn to a more detailed discussion of the situation on $\mathbb{A}^{1} / k$. For any constructible $\overline{\mathbb{Q}}_{\ell^{-}}$-sheaf $\mathcal{G}$ on $\mathbb{A}^{1} / k$, we denote by $r k(\mathcal{G})$ the generic rank of $\mathcal{G}$. For each point $\alpha \in \bar{k}$, we denote by $\mathcal{G}_{\alpha}$ its stalk at $\alpha$, and by $\operatorname{drop}_{\alpha}(\mathcal{G})$ the nonnegative integer

$$
\operatorname{drop}_{\alpha}(\mathcal{G}):=\operatorname{rk}(\mathcal{G})-\operatorname{dim}\left(\mathcal{G}_{\alpha}\right) .
$$

For each point $\alpha \in \bar{k} \cup\{\infty\}$, we denote by $\operatorname{Swan}_{\alpha}(\mathcal{G})$ the nonnegative integer which is the Swan conductor of $\mathcal{G}$ at $\alpha$ (i.e., as a representation of the inertia group $I(\alpha)$, cf. [Ka-GKM, 1.6]). For all but finitely many $\alpha \in \bar{k}$, both $\operatorname{drop}_{\alpha}(\mathcal{G})$ and $\operatorname{Swan}_{\alpha}(\mathcal{G})$ vanish. For each $\alpha \in \bar{k}$, we define the total drop (i.e., the Artin conductor) to be the sum

$$
\operatorname{Totdrop}_{\alpha}(\mathcal{G}):=\operatorname{drop}_{\alpha}(\mathcal{G})+\operatorname{Swan}_{\alpha}(\mathcal{G}) .
$$

The Euler-Poincaré formula, cf. [Ray], [Ka-GKM, 2.3.1], for a constructible $\overline{\mathbb{Q}}_{\ell}$-sheaf $\mathcal{G}$ on $\mathbb{A}^{1} / k$ asserts that

$$
\chi_{c}\left(\mathbb{A}^{1} \otimes_{k} \bar{k}, \mathcal{G}\right):=\sum_{i}(-1)^{i} \operatorname{dim} H_{c}^{i}\left(\mathbb{A}^{1} \otimes_{k} \bar{k}, \mathcal{G}\right)
$$

is given by

$$
\chi_{c}\left(\mathbb{A}^{1} \otimes_{k} \bar{k}, \mathcal{G}\right)=\operatorname{rk}(\mathcal{G})-\sum_{\alpha \in \bar{k}} \operatorname{Totdrop}_{\alpha}(\mathcal{G})-\operatorname{Swan}_{\infty}(\mathcal{G}) .
$$

In general, the cohomology groups $H_{c}^{i}\left(\mathbb{A}^{1} \otimes_{k} \bar{k}, \mathcal{G}\right)$ are finite dimensional $\overline{\mathbb{Q}}_{\ell}$-spaces, endowed with an action of $F_{r o b}$, which vanish except possibly for $0 \leq i \leq 2$. Moreover, if $\mathcal{G}$ is a middle extension (cf. below) then $H_{c}^{0}\left(\mathbb{A}^{1} \otimes_{k} \bar{k}, \bar{G}\right)$ vanishes.

Given the additive character $\psi$ of $k$, we have the Artin-Schreier sheaf $\mathcal{L}_{\psi}$ on $\mathbb{A}^{1} / k$, cf. [De-ST, 1.4.2, 1.7],[Ka-GKM, 4.3]. For any regular function $f$ on $X / k$, viewed as a morphism $f: X / k \rightarrow \mathbb{A}^{1} / k$, we have 
the pulled back Artin-Schreier sheaf $\mathcal{L}_{\psi(f)}$ on $X$. For $x \in X(E)$, and $\mathcal{F}=\mathcal{L}_{\psi(f)}$, we have

$$
\mathcal{F}(E, x)=\psi\left(\operatorname{Trace}_{E / k}(f(x)) .\right.
$$

If $r(x)$ is a one-variable rational function over $k$, we can view it as a morphism to $\mathbb{A}^{1} / k$ from the dense open set $U \subset \mathbb{A}^{1} / k$ where it is holomorphic, then form the pullback sheaf $\mathcal{L}_{\psi(r)}$ on $U$, and extend it by direct image to $\mathbb{A}^{1}$, still denoting this direct image $\mathcal{L}_{\psi(r)}$. If all the poles of $r$ in $\bar{k}$ have order prime to p, then for $x \in \mathbb{A}^{1}(E)=E$, we have

$$
\mathcal{L}_{\psi(r)}(E, x)=\psi\left(\text { Trace }_{E / k}(r(x)),\right.
$$

with the convention that $\psi(\infty)=0, \operatorname{Trace}_{E / k}(\infty)=\infty$.

Similarly, if we are given a multiplicative character $\chi$ of $k$, we have the Kummer sheaf $\mathcal{L}_{\chi}$ on $\mathbb{G}_{m} / k$, cf. [De-ST, 1.4.3, 1.7], [Ka-GKM, 4.3]. Given a nonzero $k$-polynomial in one variable $g(x)$, we can view it as a morphism to $\mathbb{G}_{m} / k$ from the open set $\mathbb{A}^{1}[1 / g] / k \subset \mathbb{A}^{1} / k$ where it is invertible, then form the pullback sheaf $\mathcal{L}_{\chi(g)}$ on $\mathbb{A}^{1}[1 / g] / k$, and extend it by direct image to $\mathbb{A}^{1}$, still denoting this direct image $\mathcal{L}_{\chi(g)}$. If the data $(\chi, g)$ satisfies the nondegeneracy condition imposed in the first example of of the Introduction, then for for $x \in \mathbb{A}^{1}(E)=E$, we have

$$
\mathcal{L}_{\chi(g)}(E, x)=\chi\left(\operatorname{Norm}_{E / k}(g(x)) .\right.
$$

Suppose we are given data $(\chi, r(x), g(x))$, such that all the poles of $r(x)$ in $\bar{k}$ have order prime to $p$, and such that at every zero $\alpha$ of $g(x)$ in $\bar{k}$ which is not a pole of $r(x), \chi^{\text {ord }}(g)$ is nontrivial. Denote by

$$
\mathcal{H}:=\mathcal{L}_{\psi(r)} \otimes \mathcal{L}_{\chi(g)}
$$

the tensor product sheaf on $\mathbb{A}^{1} / k$. Then for $x \in \mathbb{A}^{1}(E)=E$, we have

$$
\mathcal{H}(E, x)=\psi\left(\text { Trace } _ { E / k } ( r ( x ) ) \chi \left(\text { Norm }_{E / k}(g(x)) .\right.\right.
$$

Now fix an integer $d \geq 1$ prime to $p$, and consider the following two conditions on a constructible $\overline{\mathbb{Q}}_{\ell}$-sheaf $\mathcal{G}$ on $\mathbb{A}^{1} / k$ :

(1) There exists a dense open set $U \subset \mathbb{A}^{1} / k$, inclusion denoted $j: U \subset \mathbb{A}^{1} / k$, such that $\mathcal{G} \mid U_{i}$ is lisse and $\iota$-pure of weight 0 , and such that $\mathcal{G} \cong j_{\star}(\mathcal{G} \mid U)$. [If this condition holds for one dense open $U$, it holds for any dense open $U$ on which $\mathcal{G}$ is lisse. When it holds, we say that $\mathcal{G}$ is a middle extension which is $\iota$-pure of weight 0.$]$

(2) Viewing $\mathcal{G}$ as a representation of $\pi_{1}(U)$, its restriction to the inertia subgroup $I(\infty)$ has all its $\infty$-breaks $\neq d$.

For example, take the data $(\chi, r(x), g(x))$ as above, and again suppose that all the poles of $r(x)$ in $\bar{k}$ have order prime to $p$, and that 
for every zero $\alpha$ of $g(x)$ in $\bar{k}$ which is not a pole of $r(x), \chi^{\operatorname{ord}_{\alpha}(g)}$ is nontrivial. Then the sheaf

$$
\mathcal{H}:=\mathcal{L}_{\psi(r)} \otimes \mathcal{L}_{\chi(g)}
$$

is a middle extension which is $\iota$-pure of weight 0 . If in addition the order of pole at $\infty$ of $r(x)$ is $<d$, or is $>d$ and prime to $p$, then $\mathcal{H}$ has its $\infty$-break $\neq d$ as well.

Before stating the main result, we need one more definition. Given

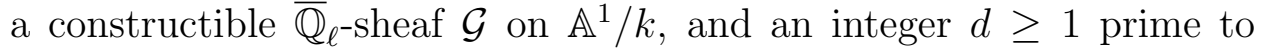
$p$, we now the nonnegative integer $\operatorname{Swan}_{d, \infty}(\mathcal{G})$ as follows. View $\mathcal{G}$ as a representation of the inertia group $I(\infty)$, and denote by $\gamma_{1}, \ldots, \gamma_{r}$, $r:=r k(\mathcal{G})$, its $\infty$-breaks. Then we define

$$
\operatorname{DiffS}_{\operatorname{Swan}}(\mathcal{G}):=\sum_{i=1}^{r}\left(\operatorname{Max}\left(d, \gamma_{i}\right)-1\right) .
$$

In the third example of the introduction, the integers $d_{i}-1$ occuring there are the integers Diff $\operatorname{Swan}_{d, \infty}\left(\mathcal{G}_{i}\right)$.

We fix an integer $d \geq 1$ prime to $p$, and a Deligne polynomial $f$ of degree $d$ in $n \geq 1$ variables over $k$. On $\mathbb{A}^{1 / k}$ we have the ArtinSchreier sheaf $\mathcal{L}_{\psi}$, and on $\mathbb{A}^{n}$, with coordinates $\left(x_{1}, \ldots, x_{n}\right)$, we have the pulled back Artin-Schreier sheaf $\mathcal{L}_{\psi\left(f\left(x_{1}, \ldots, x_{n}\right)\right)}$. For $i=1$ to $n$, we give ourselves a nonzero $\overline{\mathbb{Q}}_{\ell^{-}}$sheaf $\mathcal{G}_{i}$ on $\mathbb{A}^{1} / k$. We assume that each $\mathcal{G}_{i}$ is a middle extension which is $\iota$-pure of weight 0 , all of whose $\infty$-breaks are $\neq d$. On $\mathbb{A}^{n} / k$ we consider the tensor product sheaf

$$
\mathcal{L}_{\psi\left(f\left(x_{1}, \ldots, x_{n}\right)\right)} \otimes\left(\otimes_{i=1}^{n} \mathcal{G}_{i}\left(x_{i}\right)\right)
$$

where we denote by $\mathcal{G}_{i}\left(x_{i}\right)$ the sheaf on $\mathbb{A}^{n} / k$ which is the pullback of $\mathcal{G}_{i}$ by the $i$ 'th projection $p r_{i}: \mathbb{A}^{n} \rightarrow \mathbb{A}^{1}$.

Theorem 2.1. Hypotheses as in the preceding paragraph, we have the following results.

(1) The cohomology groups $H_{c}^{i}\left(\mathbb{A}^{n} \otimes_{k} \bar{k}, \mathcal{L}_{\psi\left(f\left(x_{1}, \ldots, x_{n}\right)\right)} \otimes\left(\otimes_{i=1}^{n} \mathcal{G}_{i}\left(x_{i}\right)\right)\right)$ vanish for $i \neq n$.

(2) The dimension of $H_{c}^{n}\left(\mathbb{A}^{n} \otimes_{k} \bar{k}, \mathcal{L}_{\psi\left(f\left(x_{1}, \ldots, x_{n}\right)\right)} \otimes\left(\otimes_{i=1}^{n} \mathcal{G}_{i}\left(x_{i}\right)\right)\right)$ is given by

$$
\operatorname{dim}_{c}^{n}=\prod_{i=1}^{n}\left(\operatorname{DiffSwan}_{d, \infty}\left(\mathcal{G}_{i}\right)+\sum_{\alpha \in \bar{k}} \operatorname{Totdrop}_{\alpha}\left(\mathcal{G}_{i}\right)\right)
$$

(3) The cohomology group $H_{c}^{n}\left(\mathbb{A}^{n} \otimes_{k} \bar{k}, \mathcal{L}_{\psi\left(f\left(x_{1}, \ldots, x_{n}\right)\right)} \otimes\left(\otimes_{i=1}^{n} \mathcal{G}_{i}\left(x_{i}\right)\right)\right)$ is ı-pure of weight $n$; i.e., via $\iota$, all the eigenvalues of Frob $k$ acting on this cohomology group have complex absolute value $(\# k)^{n / 2}$. 
By the Lefschetz Trace formula, we immediately get the following corollary, which includes the three examples of the introduction as particular cases.

Corollary 2.2. For any finite extension field $E / k$, we have the estimate

$$
\begin{aligned}
& \mid \sum_{\left(x_{1}, \ldots, x_{n}\right) \in \mathbb{A}^{n}(E)=E^{n}} \psi\left(\text { Trace }_{E / k}\left(f\left(x_{1}, \ldots, x_{n}\right)\right) \prod_{i=1}^{n} \mathcal{G}_{i}\left(E, x_{i}\right) \mid\right. \\
& \leq \prod_{i=1}^{n}\left(\operatorname{DiffSwan}_{d, \infty}\left(\mathcal{G}_{i}\right)+\sum_{\alpha \in \bar{k}} \operatorname{Totdrop}_{\alpha}\left(\mathcal{G}_{i}\right)\right)(\# E)^{n / 2} .
\end{aligned}
$$

To conclude this section, we now state a variant of the main result where we weaken slightly the hypotheses on $f$, but strengthen slightly those on the $\mathcal{G}_{i}$ 's.

Theorem 2.3. Let $d \geq 1$ be an integer which is prime to $p$. Suppose $f=f\left(x_{1}, \ldots, x_{n}\right)$ is a polynomial of degree $\leq d$ in $n$ variables (not necessarily a Deligne polynomial). If $n \geq 2$, suppose that the polynomial $f\left(x_{1}, \ldots, x_{n-1}, 0\right)$ obtained by specializing $x_{n} \mapsto 0$, is a Deligne polynomial of degree $d$ in $n-1$ variables which is Dwork-regular (as a polynomial in $n-1$ variables). Assume as above that each $\mathcal{G}_{i}$ is a middle extension which is ı-pure of weight 0 , all of whose $\infty$-breaks are $\neq d$. Assume in addition that either all the $\infty$-breaks of $\mathcal{G}_{n}$ are $>d$, or that at some point $\alpha \in \mathbb{A}^{1}(\bar{k})=\bar{k}$, the inertia group $I(\alpha)$ has no nonzero invariants acting on (the geometric generic fibre of) $\mathcal{G}_{n}$. [Because $\mathcal{G}_{n}$ is a middle extension, this second condition is the same as the condition that the stalk $\mathcal{G}_{n \alpha}=0$.] Then we have the following results.

(1) The cohomology groups $H_{c}^{i}\left(\mathbb{A}^{n} \otimes_{k} \bar{k}, \mathcal{L}_{\psi\left(f\left(x_{1}, \ldots, x_{n}\right)\right)} \otimes\left(\otimes_{i=1}^{n} \mathcal{G}_{i}\left(x_{i}\right)\right)\right)$ vanish for $i \neq n$.

(2) The dimension of $H_{c}^{n}:=H_{c}^{n}\left(\mathbb{A}^{n} \otimes_{k} \bar{k}, \mathcal{L}_{\psi\left(f\left(x_{1}, \ldots, x_{n}\right)\right)} \otimes\left(\otimes_{i=1}^{n} \mathcal{G}_{i}\left(x_{i}\right)\right)\right)$ is bounded above by

$$
\operatorname{dim}_{c}^{n} \leq \prod_{i=1}^{n}\left(\operatorname{DiffSwan}_{d, \infty}\left(\mathcal{G}_{i}\right)+\sum_{\alpha \in \bar{k}} \operatorname{Totdrop}_{\alpha}\left(\mathcal{G}_{i}\right)\right) .
$$

(3) The cohomology group $H_{c}^{n}\left(\mathbb{A}^{n} \otimes_{k} \bar{k}, \mathcal{L}_{\psi\left(f\left(x_{1}, \ldots, x_{n}\right)\right)} \otimes\left(\otimes_{i=1}^{n} \mathcal{G}_{i}\left(x_{i}\right)\right)\right)$ is ı-mixed of weight $\leq n$; i.e., via $\iota$, all the eigenvalues of Frob ${ }_{k}$ acting on this cohomology group have complex absolute value $\leq(\# k)^{n / 2}$.

(4) If the inequality in (2) is an equality, then the cohomology group $H_{c}^{n}\left(\mathbb{A}^{n} \otimes_{k} \bar{k}, \mathcal{L}_{\psi\left(f\left(x_{1}, \ldots, x_{n}\right)\right)} \otimes\left(\otimes_{i=1}^{n} \mathcal{G}_{i}\left(x_{i}\right)\right)\right)$ is ı-pure of weight $n$. 
Again from the Lefschetz Trace formula,we get the following corollary.

Corollary 2.4. In the situation of the theorem above, for any finite extension field $E / k$, we have the estimate

$$
\begin{aligned}
& \mid \sum_{\left(x_{1}, \ldots, x_{n}\right) \in \mathbb{A}^{n}(E)=E^{n}} \psi\left(\text { Trace }_{E / k}\left(f\left(x_{1}, \ldots, x_{n}\right)\right) \prod_{i=1}^{n} \mathcal{G}_{i}\left(E, x_{i}\right) \mid\right. \\
& \leq \prod_{i=1}^{n}\left(\operatorname{DiffSwan}_{d, \infty}\left(\mathcal{G}_{i}\right)+\sum_{\alpha \in \bar{k}} \operatorname{Totdrop}_{\alpha}\left(\mathcal{G}_{i}\right)\right)(\# E)^{n / 2} .
\end{aligned}
$$

\section{Proof of Theorem 2.1}

There are two cases where the theorem is already known. The case $n=1$ with arbitrary $\mathcal{G}=\mathcal{G}_{1}$ satisfying the imposed conditions (middle extension, $\iota$-pure of weight 0 , all $\infty$-breaks $\neq d$ ) is an instance of [De-Weil II, 3.2.3], with $X_{0}$ there taken to be $\mathbb{P}^{1}$. The hypothesis on $\infty$-breaks of $\mathcal{G}$ insures that the middle extension sheaf $\mathcal{L}_{\psi(f)} \otimes \mathcal{G}$ on $\mathbb{A}^{1}$ is totally wildly ramified at $\infty$, with Euler Poincaré characteristic given by

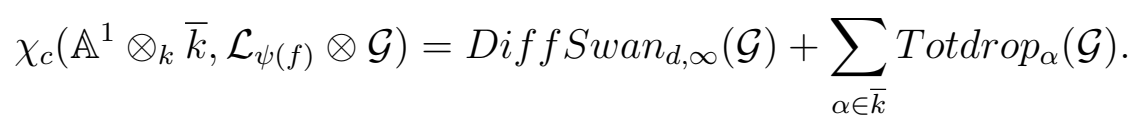

The total wildness at $\infty$ implies that $H_{c}^{i}\left(\mathbb{A}^{1} \otimes_{k} \bar{k}, \mathcal{L}_{\psi(f)} \otimes \mathcal{G}\right)$ vanishes for $i \neq 1$, and that for $j: \mathbb{A}^{1} \subset \mathbb{P}^{1}$ the inclusion, we have

$$
j_{!}\left(\mathcal{L}_{\psi(f)} \otimes \mathcal{G}\right) \cong j_{\star}\left(\mathcal{L}_{\psi(f)} \otimes \mathcal{G}\right)
$$

which in turn implies that

$$
H_{c}^{i}\left(\mathbb{A}^{1} \otimes_{k} \bar{k}, \mathcal{L}_{\psi(f)} \otimes \mathcal{G}\right) \cong H_{c}^{i}\left(\mathbb{P}^{1} \otimes_{k} \bar{k}, j_{\star}\left(\mathcal{L}_{\psi(f)} \otimes \mathcal{G}\right)\right)
$$

to which [De-Weil II, 3.2.3] applies.

The case when $n$ is arbitrary, but every $\mathcal{G}_{i}$ is the constant sheaf $\overline{\mathbb{Q}_{\ell}}$ is Deligne's theorem [De-Weil I, 8.4 and its proof], cited in the Introduction. We should emphasize here that we are not giving a new proof of that theorem, but rather using it as an essential input.

In order to prove the theorem in general, we will proceed by induction on how many of the sheaves $\mathcal{G}_{i}$ are possibly not the constant sheaf. Given integers $r \geq 0$ and $n \geq \operatorname{Max}(1, r)$, we denote by $\star(n, r)$ the statement that the theorem holds universally in $n$ variables, so long as all but at most $r$ of the sheaves $\mathcal{G}_{i}$ are the constant sheaf.

As noted above, we know that $\star(n, 0)$ holds for all $n \geq 1$, and that $\star(1,1)$ holds. 
Suppose by induction that for some $r \geq 0, \star(n, r)$ holds for all $n \geq$ $\operatorname{Max}(1, r)$. We must show that $\star(n+1, r+1)$ holds for all $n \geq r$. When we start with $r=0$, it suffices to show tha $\star(n+1,1)$ holds for all $n \geq 1$, since $\star(1,1)$ holds. So whatever the starting value of $r$, it suffices to show that $\star(n+1, r+1)$ holds for all $n \geq \operatorname{Max}(1, r)$. Renumbering the variables, we may assume that one of the $r+1$ possibly nontrivial $\mathcal{G}_{i}$ is the last one, $\mathcal{G}_{n+1}$. Thus among the $n$ sheaves $\mathcal{G}_{i}$ with $i \leq n$, at most $r$ of them are nonconstant.

We will proceed by a sheaf-theoretic incarnation of the following "finite sum" procedure. We write our $n+1$ variable sum as

$$
\begin{gathered}
\sum_{\left(x_{1}, \ldots, x_{n+1}\right) \in k^{n+1}} \psi\left(f\left(x_{1}, \ldots, x_{n+1}\right)\right) \prod_{i=1}^{n+1} \mathcal{G}_{i}\left(x_{i}\right) \\
=\sum_{\alpha \in k} \mathcal{F}(\alpha) \mathcal{G}_{n+1}(\alpha)
\end{gathered}
$$

with

$$
\mathcal{F}(\alpha):=\sum_{\left(x_{1}, \ldots, x_{n}\right) \in k^{n}} \psi\left(f\left(x_{1}, \ldots, x_{n}, \alpha\right)\right) \prod_{i=1}^{n} \mathcal{G}_{i}\left(x_{i}\right) .
$$

The point of this expression is that each sum $\mathcal{F}(\alpha)$ is covered by the $\star(n, r)$ induction hypothesis, as results from the first part of the following elementary lemma.

Lemma 3.1. Let $f=f\left(x_{1}, \ldots, x_{n+1}\right)$ be a $k$-polynomial of prime to $p$ degree $d$ in $n+1 \geq 2$ variables. Suppose that the polynomial $f\left(x_{1}, \ldots, x_{n}, 0\right)$ in $n$ variables is a Deligne polynomial which is Dwork-regular. Then we have the following results.

(1) For any $\alpha$ in $\bar{k}$, the polynomial $f\left(x_{1}, \ldots, x_{n}, \alpha\right)$ in $n$ variables is a Deligne polynomial of degree $d$ which is Dwork-regular.

(2) For all but at most finitely many values of $\lambda \in \bar{k}$, the polynomial $f_{\lambda}:=f\left(x_{1}, \ldots, x_{n+1}\right)+\lambda x_{n+1}^{d}$ in $n+1$ variables is a Deligne polynomial which is Dwork-regular.

Proof. The notion of being a Deligne polynomial, or of being a Dworkregular Deligne polynomial, concerns only the leading form of the polynomial in question. So (1) holds because the leading form of $f\left(x_{1}, \ldots, x_{n}, \alpha\right)$ is equal to the leading form of $f\left(x_{1}, \ldots, x_{n}, 0\right)$. For (2), it suffices to treat the case when $f$ is homogeneous of degree $d$. Because $d$ is prime to $\mathrm{p}$, the hypothesis that $f\left(x_{1}, \ldots, x_{n}, 0\right)$ is a Dwork-regular Deligne polynomial means precisely that the $n$ partial derivatives $x_{i} \partial f\left(x_{1}, \ldots, x_{n}, 0\right) / \partial x_{i}, i=1, \ldots, n$, have no common zeroes in $\mathbb{P}^{n-1}(\bar{k})$, or equivalently that the common zeros, call them $Z$, in 
$\mathbb{P}^{n}(\bar{k})$ of the $n$ partial derivatives $x_{i} \partial f / \partial x_{i}, i=1, \ldots, n$ are disjoint from the hyperplane $x_{n+1}=0$. Therefore the set $Z$ of common zeroes is finite, and each of its finitely many points has a unique representative of the form $\left(a_{1}, \ldots, a_{n}, 1\right)$. We must show that for all but finitely many $\lambda \in \bar{k}$, the $n+1$ partial derivatives $x_{i} \partial f_{\lambda} / \partial x_{i}, i=1, \ldots, n+1$ have no common zero in $\mathbb{P}^{n}(\bar{k})$. Now the first $n$ of these partials are equal to the $x_{i} \partial f / \partial x_{i}, i=1, \ldots, n$. So their common zeroes in $\mathbb{P}^{n}(\bar{k})$ are precisely the finite set $Z$. At each point $\left(a_{1}, \ldots, a_{n}, 1\right)$ of $Z$, the value of $x_{n+1} \partial f_{\lambda} / \partial x_{n+1}=x_{n+1} \partial f / \partial x_{n+1}+d \lambda x_{n+1}^{d}$ is $x_{n+1} \partial f / \partial x_{n+1 \mid\left(a_{1}, \ldots, a_{n}, 1\right)}+$ $d \lambda$, so the only bad $\lambda$ 's are the \#Z (not necessarily distinct) quantities $-x_{n+1} \partial f / \partial x_{n+1 \mid\left(a_{1}, \ldots, a_{n}, 1\right)} / d$.

We now give the sheaf-theoretic interpretation of the sums $\mathcal{F}(\alpha)$. For ease of later reference, we state a slightly more general result.

Lemma 3.2. Suppose given an integer $n \geq 1$, and $n$ sheaves $\mathcal{G}_{i}, i=$ $1, \ldots, n$ on $\mathbb{A}^{1} / k$, each of which is a middle extension. On $\mathbb{A}^{n}$, form their external tensor product, $\mathcal{G}(x):=\prod_{i=1}^{n} \mathcal{G}_{i}\left(x_{i}\right)$. Let $d \geq 1$ be an integer, and Poly $(n, d) / k$ the affine space of polynomials of degree $\leq d$ in $n$ variables. On $\mathbb{A}^{n} \times{ }_{k}$ Poly $(n, d)$ with coordinates $(x, f)$, we have the universal polynomial $f_{\text {univ }}$ in $n$ variables of degree $\leq d$,

$$
f_{\text {univ }}(x, f):=f(x),
$$

and the corresponding Artin-Schreier sheaf $\mathcal{L}_{\psi\left(f_{\text {univ }}\right)}$. We also have the pullback of the sheaf $\mathcal{G}(x)$ on $\mathbb{A}^{n}$ by the first projection. We form their tensor product, $\mathcal{L}_{\psi\left(f_{\text {univ }}\right)} \otimes \mathcal{G}$. By the second projection

$$
p r_{2}: \mathbb{A}^{n} \times_{k} \operatorname{Poly}(n, d) \rightarrow \operatorname{Poly}(n, d)
$$

we form the sheaves

$$
R^{i}:=R^{i} p r_{2 !}\left(\mathcal{L}_{\psi\left(f_{\text {univ }}\right)} \otimes \mathcal{G}\right)
$$

on the base Poly $(n, d)$. Let $U \subset \operatorname{Poly}(n, d)$ be a dense open set, and $N$ an integer. Suppose that for every $f \in U(\bar{k})$, we have

$$
H_{c}^{i}\left(\mathbb{A}^{n} \otimes_{k} \bar{k}, \mathcal{L}_{\psi(f)} \otimes \mathcal{G}\right)=0 \text { for } i \neq n
$$

and

$$
\operatorname{dim} H_{c}^{n}\left(\mathbb{A}^{n} \otimes_{k} \bar{k}, \mathcal{L}_{\psi(f(x))} \otimes \mathcal{G}(x)\right)=N .
$$

Then the sheaves $R^{i} \mid U$ vanish for $i \neq n$, and the sheaf $R^{n} \mid U$ is lisse on $U$ of rank $N$, with trace function on $U$ given by

$$
R^{n}(E, f)=(-1)^{n} \sum_{x \in E^{n}} \psi\left(\text { Trace }_{E / k}(f(x)) \prod_{i=1}^{n} \mathcal{G}_{i}\left(E, x_{i}\right) .\right.
$$


Proof. That the sheaves $R^{i} \mid U$ vanish for $i \neq n$ may be checked point by point, where, by proper base change, it is the hypothesis. Once $R^{n} \mid U$ is the only nonvanishing cohomology sheaf, the assertion about its trace function is just the Lefschetz Trace formula (again by proper base change). It remains to explain why $R^{n} \mid U$ is lisse. Let $m:=$ $\operatorname{dimPoly}(n, d)$. Then the shifted sheaf

$$
\mathcal{L} \psi\left(f_{\text {univ }}\right) \otimes \mathcal{G}[n+m]
$$

on $\mathbb{A}^{n} \times_{k} \operatorname{Poly}(n, d)$ is perverse, and the morphism $p r_{2}$ is affine. Therefore the sheaf $\mathcal{H}^{-m}\left(\operatorname{Rpr}_{2 !}\left(\mathcal{L} \psi\left(f_{\text {univ }}\right) \otimes \mathcal{G}[n+m]\right)\right)$ on $\operatorname{Poly}(n, d)$, which is precisely the sheaf $R^{n}$, is a sheaf of perverse origin, cf [Ka-SCMD, Introduction and Cor. 5]. One knows of [Ka-SCMD, Prop. 11] that a sheaf of perverse origin (on any geometrically connected smooth $k$ scheme) is lisse on any open set where its stalks have constant rank.

We now return to the proof of the theorem. Thus $d$ is fixed, and we assume that for a given $r \geq 0, \star(n, r)$ holds for all $n \geq \operatorname{Max}(r, 1)$. We must show that $\star(n+1, r+1)$ holds for all $n \geq \operatorname{Max}(1, r)$. We are given $n+1$ middle extension sheaves $\mathcal{G}_{i}$ on $\mathbb{A}^{1} / k$, each of which is pure of weight zero, with no $\infty$-break $=d$. Among the first $n$ sheaves $\mathcal{G}_{i}$ with $i \leq n$, at most $r$ of them are nonconstant.

Fix a $k$-polynomial $f\left(x_{1}, \ldots, x_{n+1}\right)$ of degree $\leq d$ such that $f\left(x_{1}, \ldots, x_{n}, 0\right)$ as a polynomial in $n$ variables is a Deligne polynomial of degree $d$ which is Dwork-regular. On $\mathbb{A}^{n+1} / k$, form the sheaf $\mathcal{L}_{\psi(f)} \otimes\left(\otimes_{i=1}^{n} \mathcal{G}_{i}\left(x_{i}\right)\right)$. Map $\mathbb{A}^{n+1} / k$ to $\mathbb{A}^{1} / k$ by the projection $p r_{n+1}$ onto $x_{n+1}$. We claim that the sheaves

$$
R^{i} p r_{n+1 !}\left(\mathcal{L}_{\psi(f)} \otimes\left(\otimes_{i=1}^{n} \mathcal{G}_{i}\left(x_{i}\right)\right)\right)
$$

on $\mathbb{A}^{1}$ vanish for $i \neq n$, and the remaining sheaf

$$
\mathcal{F}_{n}:=R^{n} p r_{n+1 !}\left(\mathcal{L}_{\psi(f)} \otimes\left(\otimes_{i=1}^{n} \mathcal{G}_{i}\left(x_{i}\right)\right)\right)
$$

is lisse of rank

$$
N:=\prod_{i=1}^{n}\left(\operatorname{DiffS}_{\operatorname{Swan}_{d, \infty}}\left(\mathcal{G}_{i}\right)+\sum_{\alpha \in \bar{k}} \operatorname{Totdrop}_{\alpha}\left(\mathcal{G}_{i}\right)\right) .
$$

Indeed, this follows from the previous lemma, and the induction hypothesis $\star(n, r)$; we take for $U$ in that lemma the dense open set of $\operatorname{Poly}(n, d)$ consisting of Dwork-regular Deligne polynomials, and then we pull back by the map $\mathbb{A}^{1} / k \rightarrow U$ given by $\alpha \mapsto f\left(x_{1}, \ldots, x_{n}, \alpha\right)$ to obtain the situation under discussion.

We now study the ramification at $\infty$ of the lisse sheaf $\mathcal{F}_{n}$ on $\mathbb{A}^{1} / k$. For any constructible $\overline{\mathbb{Q}}_{\ell^{-}}$sheaf $\mathcal{H}$ on $\mathbb{A}^{1} / k$, we can form the sheaf

$$
\mathcal{L}_{\psi(f)} \otimes\left(\otimes_{i=1}^{n} \mathcal{G}_{i}\left(x_{i}\right)\right) \otimes \mathcal{H}\left(x_{n+1}\right)
$$


on $\mathbb{A}^{n+1}$. By the projection formula for $p r_{n+1}$, we have $R^{i} p r_{n+1 !}\left(\mathcal{L}_{\psi(f)} \otimes\left(\otimes_{i=1}^{n} \mathcal{G}_{i}\left(x_{i}\right)\right) \otimes \mathcal{H}\left(x_{n+1}\right)\right) \cong R^{i} p r_{n+1 !}\left(\mathcal{L}_{\psi(f)} \otimes\left(\otimes_{i=1}^{n} \mathcal{G}_{i}\left(x_{i}\right)\right)\right) \otimes \mathcal{H}$

So the only nonvanishing such $R^{i}$ is $R^{n}$, and this $R^{n}$ is just the tensor product $\mathcal{F}_{n} \otimes \mathcal{H}$. So in the Leray spectral sequence for $R p r_{n+1 !}$, only the terms $E_{2}^{a, b}$ with $b=n$ are possibly nonzero, and they are given by

$$
E_{2}^{a, n}=H_{c}^{a}\left(\mathbb{A}^{1} \otimes_{k} \bar{k}, \mathcal{F}_{n} \otimes \mathcal{H}\right) .
$$

Therefore this spectral sequence degenerates at $E_{2}$. It shows that the groups

$$
H_{c}^{i}:=H_{c}^{i}\left(\mathbb{A}^{n+1} \otimes_{k} \bar{k}, \mathcal{L}_{\psi(f)} \otimes\left(\otimes_{i=1}^{n} \mathcal{G}_{i}\left(x_{i}\right) \otimes \mathcal{H}\left(x_{n+1}\right)\right)\right.
$$

vanish except possibly for $i \in\{n, n+1, n+2\}$, and it shows that for these values of $i$ we have

$$
H_{c}^{n+a} \cong H_{c}^{a}\left(\mathbb{A}^{1} \otimes_{k} \bar{k}, \mathcal{F}_{n} \otimes \mathcal{H}\right) .
$$

Passing to Euler characteristics, we obtain

$$
(-1)^{n} \chi_{c}\left(\mathbb{A}^{1} \otimes_{k} \bar{k}, \mathcal{F}_{n} \otimes \mathcal{H}\right)=\chi_{c}\left(\mathbb{A}^{n+1} \otimes_{k} \bar{k}, \mathcal{L}_{\psi(f)} \otimes\left(\otimes_{i=1}^{n} \mathcal{G}_{i}\left(x_{i}\right)\right) \otimes \mathcal{H}\left(x_{n+1}\right)\right) .
$$

Lemma 3.3. For $f$ as above (i.e., a k-polynomial $f\left(x_{1}, \ldots, x_{n+1}\right)$ of degree $\leq d$ such that $f\left(x_{1}, \ldots, x_{n}, 0\right)$ as a polynomial in $n$ variables is a Deligne polynomial of degree $d$ which is Dwork-regular), the lisse sheaf $\mathcal{F}_{n}$ has all its $\infty$-breaks $\leq d$. If $f$ is itself a Dwork-regular Deligne polynomial of degree $d$ in $n+1$ variables, then $\mathcal{F}_{n}$ has all its $\infty$-breaks $=d$.

Proof. In either case, we know that for all but finitely many $\lambda \in \bar{k}$, the polynomial $f_{\lambda}:=f\left(x_{1}, \ldots, x_{n+1}\right)+\lambda x_{n+1}^{d}$ in $n+1$ variables is a Deligne polynomial which is Dwork-regular. For any such good $\lambda$, replacing if necessary $k$ by a finite extension field containing $\lambda$, use the auxiliary sheaf $\mathcal{H}:=\mathcal{L}_{\psi\left(\lambda x^{d}\right)}$. The sheaf on $\mathbb{A}^{n+1}$ simplifies:

$$
\left.\mathcal{L}_{\psi(f)} \otimes\left(\otimes_{i=1}^{n} \mathcal{G}_{i}\left(x_{i}\right)\right) \otimes \mathcal{L}_{\psi\left(\lambda x_{n+1}^{d}\right)}\right) \cong \mathcal{L}_{\psi\left(f_{\lambda}\right)} \otimes\left(\otimes_{i=1}^{n} \mathcal{G}_{i}\left(x_{i}\right)\right),
$$

and this last sheaf comes under the aegis of the induction hypothesis $\star(n+1, r)$ applied to $f_{\lambda}$, since for it the last $\mathcal{G}_{n+1}$ is the trivial sheaf. So we we have the formula

$$
\chi_{c}\left(\mathbb{A}^{n+1} \otimes_{k} \bar{k}, \mathcal{L}_{\psi\left(f_{\lambda}\right)} \otimes\left(\otimes_{i=1}^{n} \mathcal{G}_{i}\left(x_{i}\right)\right)\right)=(-1)^{n+1}(d-1) N,
$$

and hence the formula

$$
\chi_{c}\left(\mathbb{A}^{1} \otimes_{k} \bar{k}, \mathcal{F}_{n} \otimes \mathcal{L}_{\psi\left(\lambda x^{d}\right)}\right)=-(d-1) N
$$


for each such good $\lambda$. Now $\mathcal{F}_{n}$ is lisse of rank $N$ on $\mathbb{A}^{1} \otimes_{k} \bar{k}$, as is $\mathcal{F}_{n} \otimes \mathcal{L}_{\psi\left(\lambda x^{d}\right)}$ for every $\lambda$. The Euler Poincaré formula for a lisse sheaf $\mathcal{K}$ on $\mathbb{A}^{1} \otimes_{k} \bar{k}$ (namely $\chi_{c}(\mathcal{K})=r k(\mathcal{K})-\operatorname{Swan}_{\infty}(\mathcal{K})$ ) gives

$$
\chi_{c}\left(\mathbb{A}^{1} \otimes_{k} \bar{k}, \mathcal{F}_{n} \otimes \mathcal{L}_{\psi\left(\lambda x^{d}\right)}\right)=N-\operatorname{Swan}_{\infty}\left(\mathcal{F}_{n} \otimes \mathcal{L}_{\psi\left(\lambda x^{d}\right)}\right) .
$$

Thus we find that for every good $\lambda$, we have

$$
\operatorname{Swan}_{\infty}\left(\mathcal{F}_{n} \otimes \mathcal{L}_{\psi\left(\lambda x^{d}\right)}\right)=d N=d \times r k\left(\mathcal{F}_{n}\right) .
$$

Let $\gamma_{1}, \ldots, \gamma_{N}$ be the $\infty$-breaks of $\mathcal{F}_{n}$. Then for all but finitely many $\lambda \in \bar{k}$, the $\infty$-breaks of $\mathcal{F}_{n} \otimes \mathcal{L}_{\psi\left(\lambda x^{d}\right)}$ are $\operatorname{Max}\left(\gamma_{1}, d\right), \ldots, \operatorname{Max}\left(\gamma_{N}, d\right)$, cf. [Ka-GKM, 8.5.7.1]. But the Swan conductor is the sum of the breaks, so for such $\lambda$ which is also good, we find

$$
d N=\sum_{i=1}^{N} \operatorname{Max}\left(\gamma_{N}, d\right),
$$

and this equality shows that each $\infty$-break $\gamma_{i}$ of $\mathcal{F}_{n}$ is $\leq d$.

In case $f$ itself is a Dwork-regular Deligne polynomial, then $\lambda=0$ is a good $\lambda$, and so we get

$$
\chi_{c}\left(\mathbb{A}^{1} \otimes_{k} \bar{k}, \mathcal{F}_{n}\right)=-(d-1) N=N-d N .
$$

But $N=r k\left(\mathcal{F}_{n}\right)$, so we have $\operatorname{Swan}_{\infty}\left(\mathcal{F}_{n}\right)=d N=d \times r k\left(\mathcal{F}_{n}\right)$.As each $\infty$-break $\gamma_{i}$ of $\mathcal{F}_{n}$ is $\leq d$, we must have equality: every $\gamma_{i}$ is $d$.

We can now conclude the proof of Theorem 2.1. Recall that $d$ is fixed, and we assume that for a given $r \geq 0, \star(n, r)$ holds for all $n \geq \operatorname{Max}(r, 1)$. We must show that $\star(n+1, r+1)$ holds for all $n \geq$ $\operatorname{Max}(1, r)$. We are given $n+1$ middle extension sheaves $\mathcal{G}_{i}$ on $\mathbb{A}^{1} / k$, each of which is pure of weight zero, with no $\infty$-break $=d$. Among the first $n$ sheaves $\mathcal{G}_{i}$ with $i \leq n$, at most $r$ of them are nonconstant. Taking for $\mathcal{H}$ in the discussion preceding the last lemma the sheaf $\mathcal{G}_{n+1}$, we know that the groups

$$
H_{c}^{i}:=H_{c}^{i}\left(\mathbb{A}^{n+1} \otimes_{k} \bar{k}, \mathcal{L}_{\psi(f)} \otimes\left(\otimes_{i=1}^{n+1} \mathcal{G}_{i}\left(x_{i}\right)\right)\right)
$$

vanish except possibly for $i \in\{n, n+1, n+2\}$, and that for these values of $i$ we have

$$
H_{c}^{n+a} \cong H_{c}^{a}\left(\mathbb{A}^{1} \otimes_{k} \bar{k}, \mathcal{F}_{n} \otimes \mathcal{G}_{n+1}\right) .
$$

Because $\mathcal{G}_{n+1}$ is a middle extension, and $\mathcal{F}_{n}$ is lisse, $\mathcal{F}_{n} \otimes \mathcal{G}_{n+1}$ is a middle extension, and hence its $H_{c}^{0}$ vanishes. Because no $\infty$-break of $\mathcal{G}_{n+1}$ is $d$, while every $\infty$-break of $\mathcal{F}_{n}$ is $d$, it follows that all the $\infty$ breaks of $\mathcal{F}_{n} \otimes \mathcal{G}_{n+1}$ are nonzero. Indeed, if we denote $g:=r k\left(\mathcal{G}_{n+1}\right)$ the 
generic rank of $\mathcal{G}_{n+1}$, and by $\rho_{1}, \ldots, \rho_{g}$ its $\infty$-breaks, then the $\infty$-breaks of $\mathcal{F}_{n} \otimes \mathcal{G}_{n+1}$ are the numbers $\operatorname{Max}\left(\rho_{i}, d\right)_{i=1, \ldots, g}$, each repeated

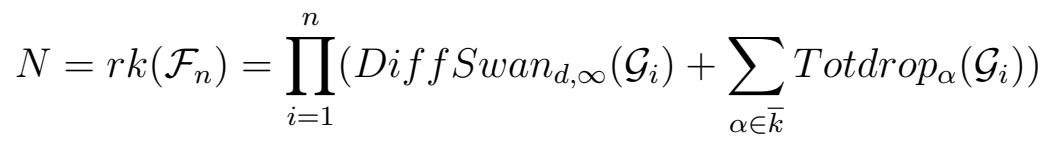

times. Because $\mathcal{F}_{n} \otimes \mathcal{G}_{n+1}$ has all its $\infty$-breaks nonzero, the inertia group $I(\infty)$ (and indeed its wild inertia subgroup $P(\infty)$ ) acts with no nonzero invariants or coinvariants, and its $H_{c}^{2}$ vanishes. And we have the formula

$$
\begin{aligned}
& \operatorname{Swan}_{\infty}\left(\mathcal{F}_{n} \otimes \mathcal{G}_{n+1}\right)-r k\left(\mathcal{F}_{n} \otimes \mathcal{G}_{n+1}\right) \\
& =N \sum_{i=1}^{g}\left(\operatorname{Max}\left(\rho_{i}, d\right)-g\right):=N \operatorname{Diff} \operatorname{Swan}_{d, \infty}\left(\mathcal{G}_{n+1}\right) .
\end{aligned}
$$

The dimension of the remaining group $H_{c}^{1}$ is then given by the Euler Poincaré formula:

$$
\begin{gathered}
-\operatorname{dimH}_{c}^{1}=\chi_{c}\left(\mathbb{A}^{1} \otimes{ }_{k} \bar{k}, \mathcal{F}_{n} \otimes \mathcal{G}_{n+1}\right) \\
=\operatorname{rk}\left(\mathcal{F}_{n} \otimes \mathcal{G}_{n+1}\right)-\sum_{\alpha \in \bar{k}} \operatorname{Totdrop}_{\alpha}\left(\mathcal{F}_{n} \otimes \mathcal{G}_{n+1}\right)-\operatorname{Swan}_{\infty}\left(\mathcal{F}_{n} \mathcal{F}_{n} \otimes \mathcal{G}_{n+1}\right) .
\end{gathered}
$$

Because $\mathcal{F}_{n}$ is lisse of rank $N$, the Euler Poincaré formula simplifies to give

$$
\begin{aligned}
-\operatorname{dimH}_{c}^{1} & =\operatorname{Nrk}\left(\mathcal{G}_{n+1}\right)-N \sum_{\alpha \in \bar{k}} \operatorname{Totdrop}_{\alpha}\left(\mathcal{G}_{n+1}\right)-N \sum_{i=1} \operatorname{Max}\left(\rho_{i}, d\right) \\
= & -N\left(\operatorname{DiffSwan}_{d, \infty}\left(\mathcal{G}_{n+1}\right)+\sum_{\alpha \in \bar{k}} \operatorname{Totdrop}_{\alpha}\left(\mathcal{G}_{n+1}\right)\right) .
\end{aligned}
$$

This shows that parts (1) and (2) of Theorem 2.1 hold. To establish (3), we argue as follows. We now apply Lemma 3.2, but with $n$ there replaced by $n+1$, with our $n+1$ sheaves $\mathcal{G}_{i}$, and with the dense open set $U \subset \operatorname{Poly}(n+1, d)$ taken to be the Dwork-regular Deligne polynomials, and with its $N$ taken to be

$$
N=\prod_{i=1}^{n+1}\left(\operatorname{DiffSwan}_{d, \infty}\left(\mathcal{G}_{i}\right)+\sum_{\alpha \in \bar{k}} \operatorname{Totdrop}_{\alpha}\left(\mathcal{G}_{i}\right)\right) .
$$

The sheaf $R^{n+1} \mid U$ is then lisse of rank $N$. It remains to show that it is $\iota$-pure of weight $n+1$ (for then specialization to our particular $f \in U(k)$ will give assertion (3)). The input sheaf

$$
\mathcal{L}_{\psi\left(f_{\text {univ }}\right)} \otimes\left(\otimes_{i=1}^{n+1} \mathcal{G}_{i}\left(x_{i}\right)\right)
$$

is certainly $\iota$-mixed, and hence by [De-Weil II, 3.3.1] the sheaf $R^{n+1}$ is $\iota$-mixed. So $R^{n+1} \mid U$ is a lisse sheaf on $U$ which is $\iota$-mixed, and hence, 
by [De-Weil II, 3.4.1], $R^{n+1} \mid U$ is a successive extension of lisse sheaves, each of which is $\iota$-pure of some weight. So to determine which weights occur, and with what multiplicities, it suffices to look at the stalk at a single point. But at any point where the variables separate, e.g., at

$f=\sum_{i=1}^{n+1} x_{i}^{d}$, the group $H_{c}^{n+1}$ is $\iota$-pure of weight $n+1$, simply because here we have a product of $n+1$ one-variable situations, each pure of weight 1.

\section{Proof of Theorem 2.3}

Let us recall the situation: $d \geq 1$ is an integer which is prime to $\mathrm{p}$, and $f=f\left(x_{1}, \ldots, x_{n}\right)$ is a polynomial of degree $\leq d$ in $n$ variables. If $n \geq 2$, we suppose that the polynomial $f\left(x_{1}, \ldots, x_{n-1}, 0\right)$ obtained by specializing $x_{n} \mapsto 0$, is a Deligne polynomial of degree $\mathrm{d}$ in $n-1$ variables which is Dwork-regular. Each $\mathcal{G}_{i}$ is a middle extension which is $\iota$-pure of weight 0 , all of whose $\infty$-breaks are $\neq d$. We assume in addition that either all the $\infty$-breaks of $\mathcal{G}_{n}$ are $>d$, or that at some point $\alpha \in \mathbb{A}^{1}(\bar{k})=(\bar{k}$, the inertia group $I(\alpha)$ has no nonzero invariants acting on (the geometric generic fibre of) $\mathcal{G}_{n}$.

We first treat the case $n=1$. So $f$ is a one-variable $k$-polynomial of degree $\leq d, \mathcal{G}_{1}$ is a middle extension sheaf as above, and we are looking at the middle extension sheaf $\mathcal{L}_{\psi(f)} \otimes \mathcal{G}_{1}$. The groups $H_{c}^{i}:=$ $H_{c}^{i}\left(\mathbb{A}^{1} \otimes_{k} \bar{k}, \mathcal{L}_{\psi(f)} \otimes \mathcal{G}_{1}\right)$ vanish for $i \neq 1$. The group $H_{c}^{0}$ vanishes because we have a middle extension sheaf on an open curve. The group $H_{c}^{2}$ vanishes because of the "extra" conditions imposed on $\mathcal{G}_{1}$. If $\mathcal{G}_{1}$ has all its $\infty$-breaks $>d$, then (as the $\infty$-break of $\mathcal{L}_{\psi(f)}$ is $\leq d$ ) the same holds for $\mathcal{L}_{\psi(f)} \otimes \mathcal{G}_{1}$ (which has the same $\infty$-breaks as $\mathbb{G}_{1}$ in this case) and hence the inertia group $I(\infty)$ has no nonzero invariants or coinvariants on $\mathcal{L}_{\psi(f)} \otimes \mathcal{G}_{1}$; this in turn forces $H_{c}^{2}$ to vanish. If some inertia group at finite distance $I(\alpha)$ acts on $\mathcal{G}_{1}$ with no nonzero invariants, then (as $\mathcal{L}_{\psi(f)}$ is lisse on $\mathbb{A}^{1}$ ) it operates on $\mathcal{L}_{\psi(f)} \otimes \mathcal{G}_{1}$ with no nonzero invariants (and hence no nonzero coinvariants), which again forces $H_{c}^{2}$ to vanish. That the remaining group $H_{c}^{1}$ is $\iota$-mixed of weight $\leq 1$ is an instance of [De-Weil II, 3.3.1].

Now with $\mathcal{G}_{1}$ fixed, let us vary $f$. We consider the universal situation, with the sheaf $\mathcal{L}_{f_{\text {univ }}(x)} \otimes \mathcal{G}_{1}(x)$ on the product space $\mathbb{A}^{1} \times \operatorname{Poly}(1, d)$ with coordinates $(x, f)$, and the second projection $p r_{2}: \mathbb{A}^{1} \times \operatorname{Poly}(1, d) \rightarrow$ $\operatorname{Poly}(1, d)$. The sheaves

$$
R^{i}:=R^{i} \operatorname{pr}_{2 !}\left(\mathcal{L}_{f_{\text {univ }}(x)} \otimes \mathcal{G}_{1}(x)\right)
$$

on $\operatorname{Poly}(1, d)$ vanish for $i \neq 1$, and $R^{1}$ is a sheaf of perverse origin, which by [De-Weil II, 3.3.1] is $\iota$-mixed of weight $\leq 1$. The generic rank 
of $R^{1}$, attained whenever $f$ has degree $d$ (i.e., is a one-variable Deligne polynomial of degree $d$ ), is

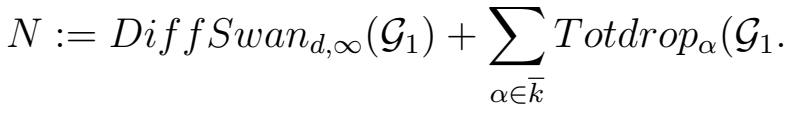

For a sheaf of perverse origin, one knows that the stalk at any point has rank at most the generic rank, and that the open set $U$ where the sheaf is lisse consists precisely of the points where the stalk has this maximum rank. This shows that for any particular $f$, we have $\operatorname{dim} H_{c}^{1} \leq N$. Moreover, if $\operatorname{dim} H_{c}^{1}=N$, then $f$ lies in $U$. The sheaf $R^{1} \mid U$ is then lisse and $\iota$-mixed, so a successive extension of pure sheaves, and we read the weights which occur from looking at any single point. Looking at $f=x^{d}$, we see from Theorem 2.1 that $H_{c}^{1}$ is pure of weight 1 , as required.

The general case is similar. For $n=m+1$ with $m \geq 1$, we proceed as in the induction step of the proof of Theorem 2.1. We consider on $\mathbb{A}^{n}=\mathbb{A}^{m+1}$ the sheaf $\mathcal{L}_{\psi(f)} \otimes\left(\otimes_{i=1}^{m} \mathcal{G}_{i}\left(x_{i}\right)\right)$, and the projection $p r_{m+1}$ : $\mathbb{A}^{m+1} \rightarrow \mathbb{A}^{1}$ onto $x_{m+1}$. Using Theorem 2.1 and the discussion following the proof of Lemma 3.2, we see that the sheaves

$$
R^{i} p r_{m+1 !}\left(\mathcal{L}_{\psi(f)} \otimes\left(\otimes_{i=1}^{m} \mathcal{G}_{i}\left(x_{i}\right)\right)\right.
$$

on $\mathbb{A}^{1}$ vanish for $i \neq m$, and the remaining sheaf

$$
\mathcal{F}_{m}:=R^{m} p r_{m+1 !}\left(\mathcal{L}_{\psi(f)} \otimes\left(\otimes_{i=1}^{m} \mathcal{G}_{i}\left(x_{i}\right)\right)\right.
$$

is lisse of rank

$$
N:=\prod_{i=1}^{m}\left(\operatorname{DiffS}_{\operatorname{Swan}_{d, \infty}}\left(\mathcal{G}_{i}\right)+\sum_{\alpha \in \bar{k}} \operatorname{Totdrop}_{\alpha}\left(\mathcal{G}_{i}\right)\right) .
$$

By Lemma 3.3 (applied with its $n$ our $m$ ), all the $\infty$-breaks of $\mathcal{F}_{m}$ are $\leq d$.The groups

$$
H_{c}^{i}:=H_{c}^{i}\left(\mathbb{A}^{1} \otimes_{k} \bar{k}, \mathcal{F}_{m} \otimes \mathcal{G}_{m+1}\right)
$$

vanish for $i \neq 1$, by exactly the same argument as in the $n=1$ case above; indeed the sheaf $\mathcal{L}_{\psi}(f)$ in the $n=1$ case plays the role of the sheaf $\mathcal{F}_{m}$ with $m=0$ in the present argument.

It remains to prove parts (2) and (4) of the theorem. The argument is similar to that given in the $n=1$ case. We fix the $n$ sheaves $\mathcal{G}_{i}$, and vary $f$. We consider the universal situation, with the sheaf $\mathcal{L}_{f_{\text {univ }}(x)} \otimes$ $\left(\otimes_{i=1}^{n} \mathcal{G}_{1}\left(x_{i}\right)\right)$ on the product space $\mathbb{A}^{n} \times \operatorname{Poly}(n, d)$ with coordinates $(x, f)$, and the second projection $p r_{2}: \mathbb{A}^{n} \times \operatorname{Poly}(1, d) \rightarrow \operatorname{Poly}(1, d)$. The sheaves

$$
R^{i}:=R^{i} \operatorname{pr}_{2 !}\left(\mathcal{L}_{f_{\text {univ }}(x)} \otimes\left(\otimes_{i=1}^{n} \mathcal{G}_{1}\left(x_{i}\right)\right)\right)
$$


on $\operatorname{Poly}(n, d)$ vanish for $i \neq n$, and $R^{n}$ is a sheaf of perverse origin, which by [De-Weil II, 3.3.1] is $\iota$-mixed of weight $\leq n$. The generic rank of $R^{n}$, attained whenever $f$ is a Dwork-regular Deligne polynomial of degree $d$, is

$$
N:=\prod_{i=1}^{n}\left(\operatorname{DiffS}_{\operatorname{Swan}_{d, \infty}}\left(\mathcal{G}_{i}\right)+\sum_{\alpha \in \bar{k}} \operatorname{Totdrop}_{\alpha}\left(\mathcal{G}_{i}\right) .\right.
$$

So at any point $f \in \operatorname{Poly}(n, d)$, the rank of $H_{c}^{n}$ is $\leq N$, and the largest open set $U \subset \operatorname{Poly}(n, d)$ on which $R^{n}$ is lisse is precisely the set of points $f$ at which $\operatorname{dim}_{c}^{n}=N$. Then $R^{n} \mid U$ is lisse and $\iota$-mixed, so a successive extension of $\iota$-pure lisse sheaves, and now we use the particular point $f=\sum_{i=1}^{n} x_{i}^{d}$ to see that $R^{n} \mid U$ is $\iota$-pure of weight $n$. This concludes the proof of Theorem 2.3.

\section{REFERENCES}

[De-ST] Deligne, P., Applications de la formule des traces aux sommes trigonométriques, pp. 168-232 in SGA 4 1/2, cited below.

[De-Weil I] Deligne, P., La conjecture de Weil. Publ. Math. IHES 43 (1974), 273307.

[De-Weil II] Deligne, P., La conjecture de Weil II. Publ. Math. IHES 52 (1981), 313-428.

[Dw-Hyp1] Dwork, B., On the Zeta Function of a Hypersurface. Publ. Math. IHES 12 (1962), 5-68.

[Gr-Rat] Grothendieck, A., Formule de Lefschetz et rationalité des fonctions $L$. Séminaire Bourbaki, Vol. 9, Exp. No. 279, 41-55, Soc. Math. France, 1995.

[Ha] Hasse, H., Theorie der relativ-zyklischen algebraischen Funktionenk'orper, insbesondere bei endlishcem Konstantk'orper. J. Reine Angew. Math. 172 (1934), $37-54$

[Ka-GKM] Katz, N., Gauss sums, Kloosterman sums, and monodromy groups, Annals of Math. Study 116, Princeton Univ. Press, 1988.

[Ka-SCMD] Katz, N., A semicontinuity result for monodromy under degeneration. Forum Math. 15 (2003), no. 2, 191-200.

[Ray] Raynaud, M., Caractéristique d'Euler-Poincaré d'un faisceau et cohomologie des variétés abéliennes. Séminaire Bourbaki, Vol. 9, Exp. No. 286, 129-147, Soc. Math. France, Paris, 1995.

[SGA 4 1/2] Cohomologie Etale. Séminaire de Géométrie Algébrique du Bois Marie SGA 4 1/2. par P. Deligne, avec la collaboration de J. F. Boutot, A. Grothendieck, L. Illusie, et J. L. Verdier. Lecture Notes in Mathematics, Vol. 569, Springer-Verlag, 1977. 
[SGA 1] Revêtements étales et groupe fondamental. Séminaire de Géométrie Algébrique du Bois Marie 1960-1961 (SGA 1). Dirigé par Alexandre Grothendieck. Augmenté de deux exposés de M. Raynaud. Lecture Notes in Mathematics, Vol. 224, Springer-Verlag, 1971.

[SGA 4 Tome 3] Théorie des Topos et Cohomologie Etale des Schémas, Tome 3. Séminaire de Géométrie Algébrique du Bois Marie 1963-1964 (SGA 4). Dirigé par M. Artin, A. Grothendieck, J. L. Verdier. Lecture Notes in Mathematics, Vol.305, Springer-Verlag, 1973.

[SGA 7 II] Groupes de monodromie en géométrie algébrique. II. Séminaire de Géométrie Algébrique du Bois-Marie 1967-1969 (SGA 7 II). Dirigé par P. Deligne et N. Katz. Lecture Notes in Mathematics, Vol. 340. Springer-Verlag, 1973.

[We] Weil, A., On some exponential sums, Proc. Nat. Acad. Sci. U. S. A. 34 (1948), 204-207.

Princeton University, Mathematics, Fine Hall, NJ 08544-1000, USA

E-mail address: nmk@math.princeton.edu 